\title{
Correction to: The Cedar Project: Using Indigenous-specific determinants of health to predict substance use among young pregnant-involved Indigenous women in Canada
}

\author{
Sana Z. Shahram ${ }^{1,8^{*}}$, Joan L. Bottorff ${ }^{2,3}$, Nelly D. Oelke ${ }^{4}$, Leanne Dahlgren ${ }^{5}$, Victoria Thomas ${ }^{6}$, Patricia M. Spittal ${ }^{7}$ \\ and For the Cedar Project Partnership
}

\section{Correction}

After publication of the original article [1] it was noted that the title of this manuscript was incorrect. The title presently reads "The cedar project: using indigenous-specific determinants of health to predict substance use among young pregnant-involved aboriginal women" but should read "The Cedar Project: Using Indigenous-specific determinants of health to predict substance use among young pregnant-involved Indigenous women in Canada".

The original manuscript was also updated.

\begin{abstract}
Author details
${ }^{1}$ Faculty of Health and Social Development, University of British Columbia, Kelowna, Canada. ${ }^{2}$ Institute for Healthy Living and Chronic Disease Prevention, and School of Nursing, Faculty of Health and Social Development, University of British Columbia, Kelowna, Canada. ${ }^{3}$ Faculty of Health Sciences, Australian Catholic University, Melbourne, Australia. ${ }^{4}$ Schoo of Nursing, Faculty of Health and Social Development, University of British Columbia, Kelowna, Canada. ${ }^{5}$ Department of Obstetrics \& Gynaecology, Faculty of Medicine, University of British Columbia, Vancouver, Canada. ${ }^{6}$ Wuikinuxv Nation, The Cedar Project, Vancouver, Canada. ${ }^{7}$ School of Population and Public Health, University of British Columbia, Vancouver, Canada. ${ }^{8}$ Present Address: Postdoctoral Research Fellow, Centre for Addictions Research of British Columbia, University of Victoria, Victoria, Canada.
\end{abstract}

Published online: 29 November 2017

\section{Reference \\ 1. Shahram SZ, Bottorff JL, Oelke ND, Dahlgren L, Thomas V, Spittal PM. The cedar project: using indigenous-specific determinants of health to predict substance use among young pregnant-involved aboriginal women. BMC Womens Health. 2017;17(1):84.}

\footnotetext{
* Correspondence: sanashahram@gmail.com

${ }^{1}$ Faculty of Health and Social Development, University of British Columbia, Kelowna, Canada

${ }^{8}$ Present Address: Postdoctoral Research Fellow, Centre for Addictions

Research of British Columbia, University of Victoria, Victoria, Canada
} 